Article

\title{
Trending Towards Safer Breast Cancer Surgeries? Examining Acute Complication Rates from A 13-Year NSQIP Analysis
}

\author{
Michael M. Jonczyk ${ }^{1,2}, * \mathbb{C}$, Jolie Jean ${ }^{3}$, Roger Graham ${ }^{1}$ and Abhishek Chatterjee ${ }^{1}$ \\ 1 Department of Surgery, Tufts Medical Center, 800 Washington Street, South Building, 4th Floor, \\ Boston, MA 02111, USA; rgraham@tuftsmedicalcenter.org (R.G.); achatterjee1@tuftsmedicalcenter.org (A.C.) \\ 2 Department of Clinical and Translational Science, Tufts University Sackler Graduate School, \\ 136 Harrison Ave \#813, Boston, MA 02111, USA \\ 3 Tufts University School of Medicine, 145 Harrison Ave, Boston, MA 02111, USA; jolie.jean@tufts.edu \\ * Correspondence: mjonczyk@tuftsmedicalcenter.org
}

Received: 24 December 2018; Accepted: 18 February 2019; Published: 21 February 2019

\begin{abstract}
As breast cancer surgery continues to evolve, this study highlights the acute complication rates and predisposing risks following partial mastectomy (PM), mastectomy $(\mathrm{M})$, mastectomy with muscular flap reconstruction $(\mathrm{M}+\mathrm{MF})$, mastectomy with implant reconstruction $(\mathrm{M}+\mathrm{I})$, and oncoplastic surgery (OPS). Data was collected from the American College of Surgeons NSQIP database (2005-2017). Complication rate and trend analyses were performed along with an assessment of odds ratios for predisposing risk factors using adjusted linear regression. 226,899 patients met the inclusion criteria. Complication rates have steadily increased in all mastectomy groups $(p<0.05)$. Cumulative complication rates between surgical categories were significantly different in each complication cluster (all $p<0.0001$ ). Overall complication rates were: PM: $2.25 \%$, OPS: $3.2 \%$, M: $6.56 \%$, M + MF: $13.04 \%$ and M + I: $5.68 \%$. The most common predictive risk factors were mastectomy, increasing operative time, ASA class, BMI, smoking, recent weight loss, history of CHF, COPD and bleeding disorders (all $p<0.001$ ). Patients who were non-diabetic, younger (age $<60$ ) and treated as an outpatient all had protective OR for an acute complication $(p<0.0001)$. This study provides data comparing nationwide acute complication rates following different breast cancer surgeries. These can be used to inform patients during surgical decision making.
\end{abstract}

Keywords: breast conservation surgery; oncoplastic surgery; mastectomy; mastectomy with reconstruction; complication rate; comorbidity; trend analysis

\section{Introduction}

Breast cancer surgery is adapting to rising patient preferences for breast reconstructive procedures. Treatment of breast cancer surgery can be classified into two overall groups: breast conserving therapy (BCT) including partial mastectomy (PM) and oncoplastic surgery (OPS), and mastectomy (MAST) including mastectomy alone (M) and $M$ with breast reconstruction (M + R). From 2005 to 2017, the use of breast reconstruction significantly increased compared to other types of breast cancer surgery for patients with both ductal carcinoma in situ (DCIS) and invasive carcinoma (IvBC) [1]. Known for its extensive use of tissue mobilization and re-arrangement to ensure optimal reshaping with breast cosmesis [2], the use of OPS (a form of breast reconstruction) has doubled from $2 \%$ to $5 \%$. In parallel, mastectomy with implant placement $(\mathrm{M}+\mathrm{I})$ increased from $11 \%$ to $21 \%$ [1]. Meanwhile, mastectomy with muscular flap reconstruction $(\mathrm{M}+\mathrm{MF})$ has actually declined from $4.5 \%$ to only $1 \%[1]$. Accompanying these changes in breast reconstruction is the steady decline of non-cosmetic procedures like traditional mastectomies (M). These shifting trends in breast cancer treatment are multifactorial 
and are likely attributed to changes in complication rates, comorbidities, patient demographics, patient surgical preference, and oncological guidelines for appropriate surgical resection [1].

Breast reconstruction (OPS or $\mathrm{M}+\mathrm{R}$ ) offers patients an improved quality of life by providing an aesthetically symmetric breast together with higher patient satisfaction [2-4]. Unfortunately, complication rates persist in breast cancer surgery and vary $(2-40 \%)$ with the type of reconstruction. They also vary depending on whether we measure short-term or long-term outcomes [2,5-13]. Fortunately, mortality in breast cancer surgery remains very low $(<1 \%)$ regardless of the type of surgery offered [14]. Post-operative complications are influenced by multiple risk factors that surgeons should consider. Wound infections and postoperative infectious complications have been associated with smoking, prior radiation, obesity and diabetes $[5,8]$.

Several studies have also shown that multiple comorbidities and higher American Society of Anesthesiologist (ASA) classification predict higher complication rates following all surgery subtypes. Nevertheless, specifically pertaining to breast cancer intervention, many patient factors and surgical predictors thought to influence acute postoperative complications are unknown or controversial [5,8,15-17]. Single institutional studies and prior reviews have generally suffered from small sample size and have lacked the power to adequately analyze the multiple variables influencing post-operative acute complications following breast cancer surgery.

Understanding surgical complications is crucial to patient safety and improving health care outcomes. Therefore, this study sought to examine the acute postoperative complication rates in breast cancer patients who underwent PM, M, M + I, M + MF, and OPS. Using the NSQIP database, we aimed to expand our understanding of predictive factors associated with different surgical procedures performed between 2005 and 2017 and evaluated trends over time.

\section{Methods and Materials}

This study follows the same methodology, inclusion/exclusion criteria, data collection and surgical categorization used in Jonczyk et al. [1]. A retrospective cohort analysis was conducted using the ACS-NSQIP database from 2005 to 2017. All participant user files (PUF) were obtained and approved by ACS NSQIP. The Institutional Review Board deemed this study exempt from institutional review given that the ACS NSQIP database is a de-identified data set.

\subsection{Data Collection}

Inclusion criteria for this study were women with classified post-operative diagnosis of invasive breast cancer (IvBC) or ductal carcinoma in-situ (DCIS) who underwent any BCT or any MAST procedure. Post-operative diagnosis was classified according to International Classification of Diseases Ninth Revision (ICD-9) code for IvBC (ICD-9, 174) or DCIS (ICD-9, 233). After October 2015, ICD Tenth Edition replaced the previous system of classification, and patients with IvBC or DCIS were classified under the appropriate ICD-10 codes: D05, D5.1-D05.99 (DCIS), and IvBC (C50). In order to examine complications for specific interventions, each surgical group (except for PM) was further divided into categories (CG) shown in Table S1. A schematic of surgical (M, PM, OPS, M + R) categorization using CPT codes is shown in Figure S1. Exclusion criteria included males, surgery for benign breast disease, lobular carcinoma in situ, patients undergoing breast cancer surgery with 2 CPT codes with ambiguous category placement and septic patients at time of surgery.

\subsection{Complications and Outcome Measures}

We identified 16 acute complications in the NSQIP database that were collected prospectively in a 30-day post-operative period. We used these complications and clustered them into eight groups based on their medical similarity. Table 1 depicts complication clustering and the individual complications included. 
Table 1. Complication Clustered from NSQIP database.

\begin{tabular}{ll}
\hline Clusters & Individual Complication with NSQIP Code \\
\hline Wound Complications & $\begin{array}{l}\text { Superficial Incisional Infection SSI: SUPINFEC } \\
\text { Deep incisional Infection DSI: WNDINFD }\end{array}$ \\
\hline \multirow{2}{*}{ Infectious Complications } & $\begin{array}{l}\text { Organ/Space SSI: ORGSPCSSI } \\
\text { Urinary Tract Infection UTI: URNINFEC } \\
\text { Sepsis: OTHSYSEP } \\
\text { Septic Shock: OTHSESHOCK }\end{array}$ \\
\hline Respiratory Complications & $\begin{array}{l}\text { Pneumonia: OUPNEUMO } \\
\text { Unplanned re-intubation: REINTUB }\end{array}$ \\
\hline Thromboembolic Complications & $\begin{array}{l}\text { Pulmonary Embolism: PULEMBOL } \\
\text { DVT requiring Therapy: OTHDVT }\end{array}$ \\
\hline Bleeding Complications & Intraoperative or post-operative transfusion: OTHBLEED \\
\hline Renal Complications & Postoperative Renal Failure: OPRENAFL \\
\hline Cardiac Complications & Progressive Renal Insufficiency: RENAFAIL \\
\hline Stroke Complications & Cardiac arrest requiring CPR: CDARREST \\
\hline
\end{tabular}

Demographics, patient comorbidities, and surgical factors were also collected for each surgical category (Table 2). Body mass index (BMI) was not included in NSQIP and was therefore calculated using weight (lbs.) divided by squared height, multiplied by 703 [13]. Each patient was categorized as: underweight (BMI < 18.5), normal (BMI 18.5-25), overweight (BMI 25-30) or obese (BMI > 30).

Table 2. Patient Demographics and Comorbidities in each Surgical Category.

\begin{tabular}{|c|c|c|c|c|c|c|c|}
\hline \multicolumn{2}{|c|}{ Demographics (\%) } & \multirow{2}{*}{$\begin{array}{c}\begin{array}{c}\text { PM } \\
n=95,468\end{array} \\
56.63\end{array}$} & \multirow{2}{*}{$\begin{array}{c}\begin{array}{c}\text { OPS } \\
n=7279\end{array} \\
54.71\end{array}$} & \multirow{2}{*}{$\begin{array}{c}\begin{array}{c}\mathbf{M} \\
n=\mathbf{7 0 , 6 1 6}\end{array} \\
42.19\end{array}$} & \multirow{2}{*}{$\begin{array}{c}\mathrm{M}+\mathrm{MF} \\
n=4747\end{array}$} & \multirow{2}{*}{$\begin{array}{c}\mathbf{M}+\mathbf{I} \\
n=44,093\end{array}$} & \multirow{2}{*}{$\begin{array}{c}p \text {-Value } \\
<0.0001\end{array}$} \\
\hline Age & Young: $<60$ & & & & & & \\
\hline & Older: $>60$ & 43.37 & 45.29 & 57.81 & 24.73 & 21.75 & \\
\hline \multirow[t]{5}{*}{ Race } & White & 72.66 & 74.91 & 68.73 & 73.98 & 76.77 & $<0.0001$ \\
\hline & Black & 10.71 & 11.84 & 11.83 & 12.79 & 7.99 & \\
\hline & Asian/Pacific & 3.88 & 4.09 & 6.13 & 3.71 & 4.39 & \\
\hline & Native & 0.50 & 0.16 & 0.72 & 0.21 & 0.22 & \\
\hline & Unknown & 12.24 & 8.98 & 12.59 & 9.31 & 10.63 & \\
\hline \multirow[t]{2}{*}{ Pathology } & Invasive & 78.46 & 81.04 & 86.58 & 78.83 & 80.29 & $<0.0001$ \\
\hline & DCIS & 21.54 & 19.10 & 13.42 & 21.17 & 19.71 & \\
\hline \multirow[t]{2}{*}{ Admission Status } & Inpatient & 7.15 & 15.11 & 51.81 & 93.66 & 61.99 & $<0.0001$ \\
\hline & Outpatient & 92.85 & 84.89 & 48.19 & 6.34 & 38.01 & \\
\hline \multirow[t]{4}{*}{ BMI } & Underweight & 2.09 & 1.36 & 2.80 & 1.18 & 2.29 & $<0.0001$ \\
\hline & Normal & 27.27 & 27.17 & 29.20 & 30.90 & 41.27 & \\
\hline & Overweight & 30.55 & 29.43 & 29.35 & 33.85 & 28.72 & \\
\hline & Obese & 40.07 & 29.43 & 38.65 & 34.02 & 27.71 & \\
\hline \multirow[t]{3}{*}{ Diabetic } & Non-Diabetic & 86.91 & 88.68 & 84.24 & 93.22 & 94.60 & $<0.0001$ \\
\hline & Diabetic-Insulin & 3.67 & 3.20 & 4.94 & 1.85 & 1.38 & \\
\hline & Diabetic-Oral & 9.42 & 8.12 & 10.82 & 4.93 & 4.02 & \\
\hline \multicolumn{2}{|c|}{ Pregnancy Status } & 0.03 & 0.00 & 0.06 & 0.04 & 0.02 & $<0.0001$ \\
\hline \multicolumn{2}{|c|}{ Steroid Use } & 1.87 & 1.91 & 2.70 & 1.56 & 1.76 & $<0.0001$ \\
\hline \multicolumn{2}{|c|}{ Prior Chemotherapy } & 0.83 & 1.20 & 3.37 & 2.53 & 1.66 & $<0.0001$ \\
\hline \multicolumn{2}{|c|}{ Prior Radiation Therapy } & 0.08 & 0.04 & 0.26 & 0.34 & 0.09 & $<0.0001$ \\
\hline \multicolumn{2}{|c|}{ Recent Weight Loss } & 0.27 & 0.34 & 0.72 & 0.40 & 0.29 & $<0.0001$ \\
\hline
\end{tabular}


Table 2. Cont.

\begin{tabular}{|c|c|c|c|c|c|c|c|}
\hline \multicolumn{2}{|c|}{ Demographics (\%) } & $\begin{array}{c}\text { PM } \\
n=95,468\end{array}$ & $\begin{array}{c}\text { OPS } \\
n=7279\end{array}$ & $\begin{array}{c}M \\
n=70,616\end{array}$ & $\begin{array}{l}M+M F \\
n=4747\end{array}$ & $\begin{array}{c}\mathrm{M}+\mathrm{I} \\
n=44,093\end{array}$ & $p$-Value \\
\hline \multicolumn{2}{|c|}{ PMH of Angina } & 0.07 & 0.07 & 0.11 & 0.04 & 0.02 & $<0.0001$ \\
\hline \multicolumn{2}{|c|}{ PMH of Hypertension } & 0.47 & 0.40 & 0.50 & 0328 & 0.24 & $<0.0001$ \\
\hline \multicolumn{2}{|c|}{$\mathrm{PMH}$ of $\mathrm{CHF}$} & 0.27 & 0.29 & 0.43 & 0.06 & 0.04 & $<0.0001$ \\
\hline \multicolumn{2}{|c|}{ PMH of Renal Failure } & 0.03 & 0.03 & 0.04 & 0.00 & 0.01 & 0.009 \\
\hline \multicolumn{2}{|c|}{ PMH of Bleeding Disorders } & 1.64 & 1.14 & 2.25 & 0.57 & 0.64 & $<0.0001$ \\
\hline \multicolumn{2}{|c|}{ PMH of COPD } & 3.05 & 1.81 & 3.96 & 0.93 & 0.80 & $<0.0001$ \\
\hline \multicolumn{2}{|c|}{ Recent Pneumonia } & 0.01 & 0.00 & 0.01 & 0.00 & 0.00 & $<0.0001$ \\
\hline \multirow[t]{3}{*}{ Dyspnea } & At Rest & 0.29 & 0.08 & 0.42 & 0.06 & 0.06 & $<0.0001$ \\
\hline & Moderate & 5.56 & 3.49 & 7.48 & 3.60 & 2.46 & \\
\hline & None & 94.15 & 96.43 & 92.11 & 96.33 & 97.48 & \\
\hline \multirow[t]{5}{*}{ ASA Class } & 1 & 6.19 & 5.19 & 3.74 & 6.38 & 7.88 & $<0.0001$ \\
\hline & 2 & 58.95 & 60.35 & 51.60 & 69.20 & 69.34 & \\
\hline & 3 & 33.25 & 33.48 & 42.22 & 24.04 & 21.69 & \\
\hline & 4 & 1.36 & 0.80 & 2.33 & 0.32 & 0.30 & \\
\hline & 5 & 0.00 & 0.00 & 0.00 & 0.00 & 0.00 & \\
\hline \multicolumn{2}{|c|}{ Open Wound Infection } & 0.37 & 0.25 & 1.31 & 1.22 & 0.20 & $<0.0001$ \\
\hline Prior Infection & SIRS & 0.20 & 0.23 & 0.42 & 0.55 & 0.23 & $<0.0001$ \\
\hline (Within 48 h) & Sepsis & 0.01 & 0.01 & 0.04 & 0.06 & 0.00 & \\
\hline \multicolumn{2}{|c|}{ Any Operation in Last 30 Days } & 1.92 & 0.91 & 1.30 & 2.19 & 0.66 & $<0.0001$ \\
\hline \multirow[t]{6}{*}{ Operating Time } & Less than $1 \mathrm{~h}$ & 0.48 & 0.16 & 0.13 & 0.01 & 0.01 & \multirow{3}{*}{$<0.0001$} \\
\hline & $1-2 \mathrm{~h}$ & 0.42 & 0.37 & 0.47 & 0.04 & 0.11 & \\
\hline & $2-3 \mathrm{~h}$ & 0.08 & 0.23 & 0.12 & 0.07 & 0.29 & \\
\hline & $3-5 \mathrm{~h}$ & 0.02 & 0.18 & 0.02 & 0.34 & 0.45 & \\
\hline & 5-10 h & 0 & 0.05 & 0 & 0.47 & 0.12 & \\
\hline & $10+h$ & 0 & 0 & 0 & 0.06 & 0 & \\
\hline Return to OR & hin 30 Days) & 0.05 & 0.04 & 0.04 & 0.08 & 0.07 & $<0.0001$ \\
\hline
\end{tabular}

\subsection{NSQIP Variable Definitions}

NSQIP defines patients at risk for bleeding due to any condition with deficiency of clotting elements (Vitamin K deficiency, hemophilia's, thrombocytopenia, or on chronic anticoagulation other than aspirin). Chemotherapy and radiation were defined as being administered pre-operatively for malignancy in less than 30 days and 90 days, respectively. Open wound infections (OWI) were any breach of skin integrity with or without cellulitis or purulent exudate when leaving the operating room and this included the use of drain devices or negative pressure wound devices. OWI did not include scabbed over wound or Band-Aid covered sores (break in skin), tracheostomy, oral sores and ostomies. For this analysis, we associated OWI with drain placement or wound vac placement. Recent weight loss was defined as greater than $10 \%$ unintentional loss of body weight. Hypertension (HTN) had to be documented, and patients had to be on medication for over 2 weeks prior to surgical intervention.

\subsection{Statistics}

All analyses were performed using R-Studio software. Chi-square tests analyses were performed for demographic and complication rate analysis. Smoothed linear regression was used to adjust a best fit line and then a non-parametric Mann- Kendall test was used to assess complication temporal trends. The variables in Table 3 were used as covariates for a stepwise logistical regression model for each clustered complication grouping. 
Table 3. Covariates used in Multivariable Logistical Regression.

\begin{tabular}{|c|c|}
\hline Surgery Type & $\mathbf{P M}{ }^{* *}, \mathrm{OPS}, \mathrm{M}, \mathbf{M}+\mathbf{R}, \mathbf{M}+\mathbf{I}$ \\
\hline Age & $\begin{array}{l}\text { Young: }<60 \\
\text { Older: }>60 * *\end{array}$ \\
\hline Race & White ${ }^{* *}$, Black, Asian, Native, Unknown \\
\hline Pathology & $\begin{array}{c}\text { Invasive Breast Cancer } \\
\text { Ductal Carcinoma in situ }\end{array}$ \\
\hline Admission Status & Inpatient ${ }^{* *}$ or Outpatient \\
\hline BMI & $\begin{array}{c}\text { Underweight } \\
\text { Normal } \\
\text { ** } \\
\text { OverweightObese }\end{array}$ \\
\hline Pregnancy Status & Yes or $\mathrm{No}^{* *}$ \\
\hline Smoking Status & Smoker or Non-Smoker ** \\
\hline Smoking Pack Per Day (PPD) & $\begin{array}{l}\text { None ** } \\
\text { 0-20 PPD } \\
\text { 21-50 PPD } \\
\text { 50-100 PPD } \\
\text { >100 PPD }\end{array}$ \\
\hline Steroid Use & Yes or $\mathrm{No}^{* *}$ \\
\hline Prior Chemotherapy & Yes or No ${ }^{* *}$ \\
\hline Prior Radiation Therapy & Yes or No ** \\
\hline Peri-Operative ASA Class & $1 * *, 2,3,4,5$ \\
\hline Operative Wound Infection & Yes or $\mathrm{No}^{* *}$ \\
\hline Any Operation in Last 30 Days & Yes or $\mathrm{No}^{* *}$ \\
\hline Recent Pneumonia & Yes or No ${ }^{* *}$ \\
\hline Diabetic & $\begin{array}{c}\text { Non-Diabetic } \\
\text { Diabetic on Insulin ** } \\
\text { Diabetic on Oral medication }\end{array}$ \\
\hline
\end{tabular}

\begin{tabular}{cc}
\hline Recent Weight Loss & Yes or $\mathrm{No}^{* *}$ \\
\hline PMH of Angina & Yes or $\mathrm{No}^{* *}$ \\
\hline PMH of Congestive Heart Failure & Yes or No ${ }^{* *}$ \\
\hline PMH of Renal Failure & Yes or No ${ }^{* *}$ \\
\hline PMH of COPD & Yes or No ${ }^{* *}$ \\
\hline History of Bleeding Disorder & Yes or No ${ }^{* *}$ \\
\hline & Less than $1 \mathrm{~h}{ }^{* *}$ \\
Operating Time & $2-3 \mathrm{~h}$ \\
& $3-5 \mathrm{~h}$ \\
& $5-10 \mathrm{~h}$ \\
& Over $10 \mathrm{~h}$
\end{tabular}

\footnotetext{
* The regression model provides odds ratios compared to a baseline covariate. The asterisks ${ }^{* *}$ is the baseline covariate.
}

Each covariate was analyzed for significance in a univariate logistical regression $(p<0.05)$ for each clustered complication. All significant covariates were used in a multivariable logistical regression (MLR) to calculate the adjusted odds ratio (OR) for acquiring each complication. Each covariate was compared to its baseline covariate and a computed OR predicts an association to the baseline covariate. Patient baseline covariates used in the MLR were as follows: diabetes treated with IV insulin, white race, non-smokers and no prior smoking pack-years, older age (>60 years old), pathology (DCIS), 
normal BMI, no PMH of bleeding disorder, renal failure, angina, CHF, HTN, or COPD, and no recent weight loss. Surgical baseline covariates used in MLR were as follows: inpatient admission status, ASA class 1 , operative time $<1 \mathrm{~h}$, surgery $(\mathrm{PM})$, no open wound infection, and no prior operation within 30 days. All results were considered significant at $p$ values $<0.05$ level.

\section{Results}

\subsection{Participant Pool and Demographics}

Between 2005 and 2017, over 6 million patients were included in the NSQIP database and roughly 300,000 patients underwent breast cancer surgery. A total of 226,899 (77.9\%) women met our inclusion criteria for the present analysis (Table S2). Demographics, patient comorbidities, and surgical factors were significantly different $(<0.001)$ among all five groups (Table 2$)$. Compared to other groups, the $\mathrm{M}+\mathrm{I}$ group had the highest incidence of younger patients and a lower incidence of the following preoperative indicators: lower ASA class, fewer open wound infections or systemic infections within 48 hours of surgery, and fewer prior operations in the last 30 days.

\subsection{Overall Complication Rate and Trend Analysis}

Cumulative complication rates were analyzed between 2005-2017 and clustered into their appropriate complication groups for each surgical intervention (Table 4).

All complication clusters were significant $(<0.0001)$. The overall complication rates per surgical intervention were as follows: PM 2.25\%, OPS 3.2\%, M 6.56\%, M + MF $13.04 \%$ and M + I 5.68\%. Wound, infectious, respiratory, bleeding and thromboembolic complications were highest in the $\mathrm{M}+\mathrm{MF}$ group. The $\mathrm{M}$ group had the highest rates of renal, cardiac and stroke complications. Table 5 and Figure 1 depict the 13-year adjusted smoothed trend analysis in nationwide breast cancer surgeries.

Table 4. Complication rate in Surgical interventions.

\begin{tabular}{|c|c|c|c|c|c|c|c|c|c|c|c|}
\hline \multirow{3}{*}{$\begin{array}{l}\begin{array}{l}\text { Categories } \rightarrow \\
\text { Complication }(n, \%)\end{array} \\
\text { Wound } \\
\text { Complication }\end{array}$} & \multicolumn{4}{|c|}{ BCT $n=102,747$} & \multicolumn{6}{|c|}{ MAST $n=119,456$} & \multirow{3}{*}{$\begin{array}{l}p \text {-Value } \\
<0.0001\end{array}$} \\
\hline & \multicolumn{2}{|c|}{ PM $n=95,468$} & \multicolumn{2}{|c|}{ OPS $n=7279$} & \multicolumn{2}{|c|}{$\mathrm{M} n=70,616$} & \multicolumn{2}{|c|}{$\mathrm{M}+\mathrm{MF} n=4747$} & \multicolumn{2}{|c|}{$\mathrm{M}+\mathrm{I} n=44,093$} & \\
\hline & 1341 & 1.40 & 128 & 1.76 & 2385 & 3.38 & 244 & 5.14 & 1325 & 3.01 & \\
\hline Infectious & 456 & 0.48 & 50 & 0.69 & 823 & 1.17 & 92 & 1.94 & 652 & 1.48 & $<0.0001$ \\
\hline Respiratory & 90 & 0.09 & 8 & 0.11 & 172 & 0.24 & 12 & 0.25 & 43 & 0.10 & $<0.0001$ \\
\hline Bleeding & 66 & 0.07 & 28 & 0.38 & 844 & 1.20 & 206 & 4.34 & 277 & 0.63 & $<0.0001$ \\
\hline Thromboembolic & 99 & 0.10 & 9 & 0.12 & 200 & 0.28 & 55 & 1.16 & 179 & 0.41 & $<0.0001$ \\
\hline Renal & 21 & 0.02 & 2 & 0.03 & 53 & 0.08 & 3 & 0.06 & 11 & 0.02 & $<0.0001$ \\
\hline Cardiac & 42 & 0.04 & 4 & 0.05 & 89 & 0.13 & 3 & 0.06 & 8 & 0.02 & $<0.0001$ \\
\hline Stroke & 29 & 0.03 & 4 & 0.05 & 64 & 0.09 & 4 & 0.08 & 10 & 0.02 & $<0.0001$ \\
\hline $\begin{array}{l}\text { Overall } \\
\text { Complication Rate }\end{array}$ & 2144 & $2.25 \%$ & 233 & $3.2 \%$ & 4630 & $6.56 \%$ & 619 & $13.04 \%$ & 2505 & $5.68 \%$ & $<0.0001$ \\
\hline
\end{tabular}

BCT. Breast conservation therapy; MAST: Mastectomy group; PM: Partial Mastectomy; OcPs: Oncoplastic Surgery; M: Mastectomy; +MF: Mastectomy with Muscular Flap reconstruction; M + I: Mastectomy with Implant placement.

Table 5. Trend of Surgical Complication Rates $(n, \%) *$

\begin{tabular}{|c|c|c|c|c|c|c|c|c|c|c|}
\hline \multirow{3}{*}{$\begin{array}{l}\text { Year } \\
2005\end{array}$} & \multicolumn{10}{|c|}{ Trend of Surgical Complication Rates $(n, \%) *$} \\
\hline & \multicolumn{2}{|c|}{ PM } & \multicolumn{2}{|c|}{ OPS } & \multicolumn{2}{|c|}{$\mathbf{M}$} & \multicolumn{2}{|c|}{$\mathbf{M}+\mathbf{I}$} & \multicolumn{2}{|c|}{$\mathbf{M}+\mathbf{M F}$} \\
\hline & 22 & $2.7 \%$ & 1 & $2.8 \%$ & 32 & $5.4 \%$ & 11 & $5.5 \%$ & 4 & $7.4 \%$ \\
\hline 2006 & 68 & $2.5 \%$ & 3 & $1.6 \%$ & 112 & $5.5 \%$ & 30 & $5.6 \%$ & 17 & $7.4 \%$ \\
\hline 2007 & 103 & $2.4 \%$ & 1 & $2.7 \%$ & 193 & $5.6 \%$ & 73 & $5.1 \%$ & 24 & $8.5 \%$ \\
\hline 2008 & 110 & $2.3 \%$ & 7 & $3.7 \%$ & 254 & $5.9 \%$ & 91 & $5.0 \%$ & 38 & $8.4 \%$ \\
\hline 2009 & 119 & $2.2 \%$ & 11 & $3.7 \%$ & 326 & $6.4 \%$ & 119 & $5.1 \%$ & 35 & $10.7 \%$ \\
\hline
\end{tabular}


Table 5. Cont.

\begin{tabular}{|c|c|c|c|c|c|c|c|c|c|c|}
\hline \multirow{3}{*}{$\begin{array}{l}\text { Year } \\
2010\end{array}$} & \multicolumn{10}{|c|}{ Trend of Surgical Complication Rates $(n, \%)$ * } \\
\hline & \multicolumn{2}{|c|}{ PM } & \multicolumn{2}{|c|}{ OPS } & \multicolumn{2}{|c|}{$\mathbf{M}$} & \multicolumn{2}{|c|}{$\mathbf{M}+\mathbf{I}$} & \multicolumn{2}{|c|}{$\mathbf{M}+\mathbf{M F}$} \\
\hline & 115 & $2.1 \%$ & 6 & $3.6 \%$ & 340 & $6.9 \%$ & 147 & $5.6 \%$ & 54 & $13.2 \%$ \\
\hline 2011 & 129 & $2.1 \%$ & 15 & $3.2 \%$ & 331 & $7.0 \%$ & 192 & $6.0 \%$ & 64 & $16.0 \%$ \\
\hline 2012 & 151 & $2.2 \%$ & 16 & $3.1 \%$ & 359 & $6.8 \%$ & 224 & $5.8 \%$ & 58 & $16.5 \%$ \\
\hline 2013 & 177 & $2.1 \%$ & 16 & $2.7 \%$ & 391 & $6.6 \%$ & 249 & $5.6 \%$ & 56 & $16.8 \%$ \\
\hline 2014 & 199 & $2.3 \%$ & 21 & $3.1 \%$ & 422 & $6.7 \%$ & 252 & $5.5 \%$ & 54 & $16.7 \%$ \\
\hline 2015 & 276 & $2.3 \%$ & 37 & $3.1 \%$ & 436 & $6.9 \%$ & 280 & $5.7 \%$ & 47 & $14.7 \%$ \\
\hline 2016 & 278 & $2.3 \%$ & 33 & $3.4 \%$ & 494 & $6.8 \%$ & 320 & $5.9 \%$ & 34 & $13.8 \%$ \\
\hline 2017 & 295 & $2.2 \%$ & 45 & $3.2 \%$ & 434 & $6.8 \%$ & 340 & $6.1 \%$ & 41 & $13.3 \%$ \\
\hline \multicolumn{11}{|c|}{ Overall Trend Analysis } \\
\hline$p$-Value $\quad\left(\mathrm{R}^{2}\right)$ & 0.29 & $(0.27)$ & 0.67 & $(0.14)$ & 0.004 & $(0.71)$ & 0.02 & $(0.39)$ & 0.01 & $(0.60)$ \\
\hline
\end{tabular}

* Percentages are taken from smoothed data analysis to assess trend.

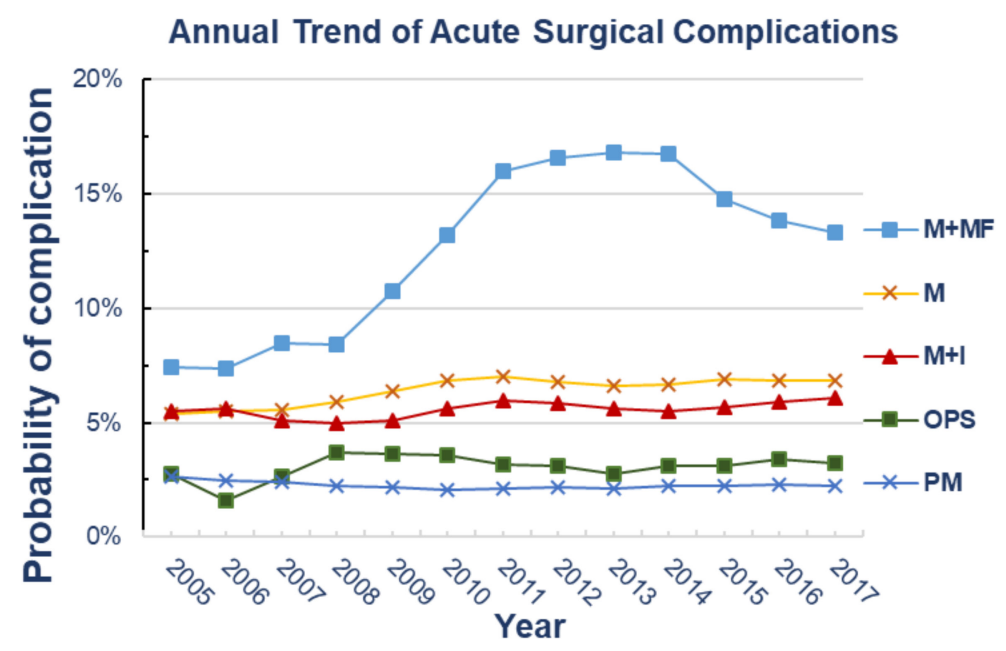

Figure 1. Annual trend analysis showing complication rate within each surgical category. PM: Partial Mastectomy; OPS: Oncoplastic Surgery; M: Mastectomy; M + MF: Mastectomy with Muscular Flap reconstruction; M + I: Mastectomy with Implant placement.

From 2005 to 2017, there was no significant trend change for acute postoperative complications in all patients in the BCT group (PM and OcPs); $(p>0.05)$. However, all categories in the MAST group had increased trends for complication rates as follows: M category $5.4 \%$ to $6.8 \%(p=0.004), \mathrm{M}+\mathrm{I} 5.5 \%$ to $6.1 \%(p=0.02)$ and $\mathrm{M}+\mathrm{MF} 7.4 \%$ to $13.3 \%(p=0.01)$.

\subsection{Independent Factors Associated with Complications $(p<0.05)$}

Factors in the unadjusted analysis most likely associated with any complication were: outpatient surgery, increased BMI, smoking, M + R, OPS, ASA Class, increasing operative time, PMH of diabetics, renal failure, angina, $\mathrm{CHF}, \mathrm{COPD}$ and HTN. Factors analyzed that were least likely to be associated with any cluster of complications included: pathology of the cancer, use of chemotherapy, PMH of dyspnea, angina or renal failure, prior operation in the last 30 days, pregnancy and recent pneumonia within $48 \mathrm{~h}$. Unadjusted OR can be seen in Table S3A-C, and significant covariates were used in the multiple linear regression (MLR). Tables 6 and 7 show MLR analysis of predictive factors associated with patient characteristics and surgical predictors, respectively. 
Table 6. Multivariable Logistical Regression Analysis of 30-Day Complication Rate: Patient Characteristics and Demographic Predictors.

\begin{tabular}{|c|c|c|c|}
\hline Complication & OR (95\% CI) & Complication & OR (95\% CI) \\
\hline Wound Complications & & \multicolumn{2}{|l|}{ Infectious Complications } \\
\hline Protective & $0.75(0.63-0.88)^{* *}$ & \multirow{4}{*}{$\begin{array}{l}\text { Protective } \\
\text { Diabetic on Oral medication } \\
\text { Non-Diabetic } \\
\text { Risk }\end{array}$} & $0.74(0.56-0.97) *$ \\
\hline Diabetic on Oral medication & $0.61(0.53-0.70)^{* * *}$ & & $0.62(0.49-0.78)^{* * *}$ \\
\hline Non-Diabetic & & & \\
\hline Risk & & & \\
\hline & & \multirow{2}{*}{$\mathrm{PMH}$ of $\mathrm{CHF}$} & $2.13(1.17-3.89) *$ \\
\hline PMH of Angina & $2.22(1.24-3.98)^{* *}$ & & $1.34(1.02-1.76)$ * \\
\hline PMH of COPD & $1.29(1.09-1.53) * *$ & PMH of COPD & $173(1.27-235) * *$ \\
\hline $\mathrm{PMH}$ of Bleeding Disorder & $1.44(1.17-1.77) * *$ & PMH of Bleeding Disorder & $1.73(1.27-2.35)^{* *}$ \\
\hline Smoker & $1.61(1.46-1.77)^{* * *}$ & PMH of HTN & $1.20(1.06-1.37)^{* *}$ \\
\hline BMI Obese & $2.17(1.97-2.38)^{* * *}$ & \multirow{2}{*}{$\begin{array}{l}\text { BMI: Overweight } \\
\text { BMI ObeseRecent Weight Loss }\end{array}$} & $1.36(1.16-1.63)^{* *}$ \\
\hline BMI: Overweight & $1.34(1.21-1.48) * * *$ & & $1.62(1.39-1.90)^{* * *}$ \\
\hline Respiratory Complications & & \multirow{2}{*}{\multicolumn{2}{|c|}{ Bleeding Complications }} \\
\hline Protective & & & \\
\hline Protective & $0.66(0.47-0.93)^{*}$ & \multirow{3}{*}{\multicolumn{2}{|c|}{$\begin{array}{l}\text { Protective } \\
\text { Non-Diabetic } \\
\text { Risk }\end{array}$}} \\
\hline Age $<60$ & & & \\
\hline Risk & & & \\
\hline PMH of COPD & $1.80(1.14-2.85) *$ & Recent Weight Loss & $1.96(1.02-3.79) *$ \\
\hline BMI: underweight & $2.25(1.14-4.42) *$ & PMH of CHF & $2.97(1.67-5.26) *$ \\
\hline Recent Weight Loss & $2.76(1.09-6.99) *$ & \multirow{2}{*}{$\begin{array}{c}\text { Steroid Use } \\
\text { PMH of Bleeding Disorder }\end{array}$} & $1.82(1.26-2.62) * *$ \\
\hline PMH of Bleeding Disorder & $2.70(1.67-4.36)^{* * *}$ & & $2.62(1.90-3.62) * * *$ \\
\hline & & \multirow{4}{*}{\multicolumn{2}{|c|}{$\begin{array}{l}\text { Thromboembolic Complications } \\
\text { Risk } \\
\text { BMI: Overweight } \\
\text { BMI: Obese }\end{array}$}} \\
\hline Neuro Complications & $014(0 \Omega 6-032) * * *$ & & \\
\hline Protective & $0.14(0.06-0.32)^{* * *}$ & & \\
\hline Age $<60$ & & & \\
\hline Renal Complications & & \multicolumn{2}{|l|}{ Cardiac Complications } \\
\hline Protective & $0.35(0.18-0.71)^{* *}$ & \multirow{3}{*}{$\begin{array}{l}\text { Protective } \\
\text { Age }<60 \\
\text { Risk }\end{array}$} & $0.53(0.30-0.91) *$ \\
\hline Non-Diabetic & & & \\
\hline Risk & & & \\
\hline PMH of Bleeding Disorder & $2.50(1.05-5.94) *$ & \multirow{4}{*}{$\begin{array}{l}\text { BMI: Underweight } \\
\text { PMH of Angina } \\
\text { PMH of HTN }\end{array}$} & \multirow{4}{*}{$\begin{array}{l}2.94(1.23-7.03) * \\
5.20(1.20-22.46) * \\
2.34(1.33-4.12) * *\end{array}$} \\
\hline PMH of Renal Failure & $10.45(1.19-91.74)$ * & & \\
\hline $\mathrm{PMH}$ of $\mathrm{CHF}$ & $5.06(1.64-15.59)^{* *}$ & & \\
\hline Recent Weight Loss & $8.39(2.87-24.53)$ ** & & \\
\hline
\end{tabular}

$p$-values: ${ }^{*} p<0.05 ;{ }^{* *} p<0.01-0.001 ;{ }^{* * *} p<0.0001$. 
Table 7. Multivariable logistical Regression Analysis of 30-Day Complication Rate: Surgical Predictors of Morbidity.

\begin{tabular}{|c|c|c|c|c|c|}
\hline Complication & OR $(95 \% \mathrm{CI})$ & Complication & OR $(95 \% \mathrm{CI})$ & Complication & OR $(95 \% \mathrm{CI})$ \\
\hline Wound Complications & & Infectious Complications & & Bleeding Complications & \\
\hline Protective & & Protective & & Protective & \\
\hline Outpatient & $0.84(0.77-0.91)^{* * *}$ & Outpatient & $0.74(0.64-0.85)^{* * *}$ & Outpatient & $0.20(0.16-0.6)^{* * *}$ \\
\hline Risk & & Risk & & Risk & \\
\hline $\mathrm{M}$ & $1.79(1.62-1.98)^{* * *}$ & $\mathrm{M}$ & $1.78(1.48-2.13)^{* * *}$ & OPS & $2.78(1.20-6.45)^{*}$ \\
\hline$M+M F$ & $2.24(1.83-2.74)^{* * *}$ & $M+M F$ & $2.24(1.60-3.13)^{* * *}$ & $M+I$ & $3.61(2.28-5.73) * * *$ \\
\hline$M+I$ & $1.72(1.51-1.96)^{* * *}$ & $M+I$ & $2.17(1.73-2.72)^{* * *}$ & $\mathrm{M}$ & $5.78(3.83-8.72)^{* * *}$ \\
\hline ASA2 & $1.22(1.02-1.48)^{*}$ & ASA2 & $1.66(1.14-2.40)^{* *}$ & $M+M F$ & $10.99(6.73-17.90)^{* * *}$ \\
\hline ASA3 & $1.57(1.29-1.91)^{* * *}$ & ASA3 & $2.44(1.67-3.58)^{* * *}$ & ASA3 & $2.18(1.41-3.37)^{* *}$ \\
\hline ASA4 & $1.67(1.23-2.26)^{* *}$ & ASA4 & $4.09(2.50-6.67)^{* * *}$ & ASA4 & $5.32(3.12-9.08) * * *$ \\
\hline Open Wound & $1.83(1.40-2.39) * * *$ & Open Wound & $1.98(1.33-2.94)^{* *}$ & Open Wound & $2.09(1.36-3.21)^{* *}$ \\
\hline OPT1 & $1.32(1.18-1.48)^{* * *}$ & OPT2. & $1.36(1.09-1.71)^{*}$ & OPT4. & $2.92(1.99-4.28) * * *$ \\
\hline OPT2 & $1.51(1.33-1.72)^{* * *}$ & OPT3. & $1.70(1.34-2.17)^{* * *}$ & OPT5. & $7.21(4.22-12.33)^{* * *}$ \\
\hline OPT3 & $1.75(1.52-2.02)^{* * *}$ & OOPT4. & $2.55(1.90-3.42)^{* * *}$ & & \\
\hline OPT4 & $2.14(1.78-2.57)^{* * *}$ & OPT5. & $2.64(1.30-5.37) *$ & & \\
\hline OPT5 & $2.81(1.85-4.27)^{* * *}$ & & & & \\
\hline Thromboembolic Complications & & Cardiac Complications & & $\begin{array}{l}\text { Respiratory } \\
\text { Complications }\end{array}$ & \\
\hline Protective & & Protective & & Protective & \\
\hline Outpatient & $0.70(0.52-0.95)^{*}$ & Outpatient & $0.20(0.11-0.35)^{* * *}$ & Outpatient & $0.24(0.16-0.35) * * *$ \\
\hline Risk & & $M+I$ & $0.17(0.04-0.83) *$ & Risk & \\
\hline $\mathrm{M}$ & $2.04(1.37-3.02)^{* *}$ & OPT1 & $0.59(0.35-0.97) *$ & ASA4 & $6.62(2.14-20.48)^{* *}$ \\
\hline $\mathrm{M}+\mathrm{MF}$ & $3.91(2.16-7.07) * * *$ & OPT2 & $0.47(0.24-0.93) *$ & & \\
\hline $\mathrm{M}+\mathrm{I}$ & $2.15(1.33-3.47)^{* *}$ & OPT3 & $0.28(0.09-0.86)^{*}$ & & \\
\hline Prior operation within 30 days & $3.20(1.97-5.20)^{* * *}$ & Risk & & & \\
\hline OPT3 & $1.95(1.16-3.27)^{*}$ & ASA4 & $10.31(1.23-86.12) *$ & & \\
\hline OPT4 & $3.40(1.89-6.09) * * *$ & & & & \\
\hline OPT5 & $6.08(2.41-15.32)^{* *}$ & Renal Complications & & Neuro Complications & \\
\hline
\end{tabular}


Table 7. Cont

\begin{tabular}{|c|c|c|c|c|c|}
\hline \multirow[t]{7}{*}{ Complication } & OR $(95 \% \mathrm{CI})$ & Complication & OR $(95 \% \mathrm{CI})$ & Complication & OR $(95 \% \mathrm{CI})$ \\
\hline & & Protective & & Protective & \\
\hline & & Outpatient & $0.26(0.13-0.52)^{* *}$ & Outpatient & $0.45(0.26-0.81)^{* *}$ \\
\hline & & & & OPT2 & $0.29(0.12-0.71)^{* *}$ \\
\hline & & & & Risk & \\
\hline & & & & OPS & $4.58(1.28-16.35)$ * \\
\hline & & & & M & $2.61(1.31-5.21)^{* *}$ \\
\hline
\end{tabular}

-values. ${ }^{*} p<0.05 ;{ }^{* *} p<0.01-0.001 ;{ }^{* * *} p<0.0001$; ASA2: Class 2 Mild Disease, ASA 3: Class 3 Severe Disease, ASA4: Class 4 Severe Life Threatening; Operative time: OPT1: 1-2 h; OPT2: 2-3 h; OPT:3: 3-5 h; OPT4: 5-10 h; OPT5: $>10 \mathrm{~h}$. 


\subsection{Adjusted Predictive Factors}

\subsubsection{Predicting Factors Associated with Lower Complication Rates}

Complications had a lower associated risk when post-operatively treated as outpatients (decrease risk ranging from $16 \%$ to $87 \%$ ) when compared to inpatients $(p<0.03)$. Compared to diabetics on insulin therapy, diabetics on oral medication were $25 \%$ less likely to acquire wound complications and had a $26 \%$ lower incidence of other infections $(p=0.005 ; p<0.02)$. Likewise, non-diabetics reduced their odds of wound complications by $40 \%$, infections by $41 \%$, bleeding complications requiring transfusions by $45 \%$, and renal complications by $67 \%(p<0.004)$. Younger patients $(<60$ years of age) were $47 \%$ less likely to have cardiac complications $(p=0.02), 39 \%$ less likely to acquire respiratory complications, and had a $67 \%$ lower risk for stroke.

\subsubsection{Predicting Factors Associated with Higher Complication Rates}

Smokers had a $1.6 \times$ higher odds of wound complications and $1.2 \times$ higher risk for infectious complications $(p<0.03)$. Compared to patients with a normal BMI, increasing BMI (obese and overweight patients) correlated with more complications. Obese patients were more likely to have wound complications by a factor of 2.2 , infections by a factor of 1.68 , respiratory complications by a factor of 1.5, and thromboembolic complications by a factor of 1.66 (all $p<0.03$ ). However, underweight BMI patients had an increased risk of respiratory $(2.22 \times)$ and cardiac $(2.83)$ complications (both $p<0.2)$. Preoperative unintentional weight loss was associated with increased infections by a factor of 3.46 ( $p$ $=0.04)$ and respiratory complication by a factor of $2.7(p=0.04)$. Steroidal use was associated with increased bleeding risk by a factor of $1.82(p=0.001)$.

Comorbidities correlated to an increased risk for five complication clusters: wound, infection, respiratory, bleeding and renal. COPD predicted a higher risk of wound complications by $1.29 \times$ $(p=0.003)$, infections by $1.36 \times(p=0.02)$ and respiratory complication by $1.81 \times(p=0.007)$. CHF increased odds of bleeding complications 3-fold $(p=0.0001)$ and renal complications 5-fold $(p=0.004)$. PMH of angina was associated with a two-fold risk of wound complications $(p=0.003)$ and cardiac complications risk $5.20 \times(p=0.03)$. Hypertensive patients on medication were more likely to acquire infections by a factor of $1.2 \times(p=0.005)$ and cardiac complications by a factor of $2.3 \times(p=0.003)$. Patients were $10 \times$ more likely to have renal complication when having a PMH of renal failure $(p=0.03)$. Similarly, PMH of a bleeding disorder was associated with a two-fold odds of bleeding complications $(<0.0001)$, respiratory compromise $(<0.0001)$, infections $(p=0.0006)$ and renal complications $(p=0.03)$.

Perioperative surgical predictors were notable for an associated trend in numerous covariate categories. Overall, all MAST procedures were almost twice as likely to suffer from wound, infectious, bleeding, thromboembolic and neurological complications $(p<0.002)$. Of note, $\mathrm{M}+\mathrm{MF}$ was a particularly significant risk factor for predicting complications. There was a 10-fold increase for bleeding complications in the $\mathrm{M}+\mathrm{MF}$ group $(<0.0001)$. A trend correlating increase in operative time was associated with increasing wound complications by $1.32-2.79 \times(p<0.0001)$, infections $1.34-2.55 \times$ $(p<0.01)$, bleeding complications 2.9-7.16 $\times(p<0.0001)$ and thromboembolic complications $1.95-6.36 \times$ $(p<0.0001)$. Compared to surgical operative times of less than $1 \mathrm{~h}$, operative times between 1 and 5 hours lowered the likelihood of cardiac complications by $43-73 \%$ and risk of stroke by $70 \%$ in some women. Rising ASA class $2-4$ correlated with increased likelihood of acquiring a wound complications $(p<0.02)$ and infections $(p<0.004)$.

\section{Discussion}

The incidence of breast cancer in the United States continues to increase. Therefore, it has become increasingly important to address the complication rates resulting from unique patient demographics and comorbidities [18]. In 1998, the Women's Health and Cancer Rights Act (WHCRA) offered patients protection and insurance coverage for reconstructive breast intervention following a mastectomy [19]. Since then, reconstructive rates have increased dramatically. With this rise in breast reconstruction, 
studies outlining the risk factors associated with these modern surgeries have become a significant part of the surgical decision making process. To our knowledge, our study is the largest analysis of surgical trends with acute post-operative complications in all breast cancer interventions in context to IvBC and DCIS.

Our data analysis shows no overall complication trends in patients undergoing BCT. However, MAST procedures all have increasing trends for complication rates and $M+M F$ had the highest complication rate in the majority of complication clusters. The diminishing use of $\mathrm{M}+\mathrm{MF}$ may be the result of its prolonged operative time and higher acute complication rate. Within the MAST group, $\mathrm{M}+\mathrm{I}$ had the lowest overall complication rate, even when compared to $\mathrm{M}$ alone. This is possibly attributed to its use in healthier patients as seen in patient demographics (Table 2). Compared to previous single institution analyses, our data falls within previously described ranges of early or acute post-operative complications rates ranging as follows: PM 1.2-1.4\% [20,21], OPS 4.8-20\% [16,20-23], M $6 \%$ [14], M + I 4-40\% [6,8,24], M + MF 16-23.7\% [5]. Varying with the type of reconstructive procedure, long-term complication rates ( $>30$ days) were typically higher $[6,9,22,24,25]$. In our data, wound complications and infections represented the majority of overall complication rates in all surgical categories. Bleeding complications requiring transfusions were the third most common complication. The occurrence of a bleeding complication was $4.34 \%$ in $\mathrm{M}+\mathrm{MF}$, followed by $1.2 \%$ in $\mathrm{M}, 0.63 \%$ in $\mathrm{M}+$ I and $0.38 \%$ in OPS.

Overall, our analysis relates to and expands on previously published data demonstrating that patients undergoing OPS had the lowest complication rates when compared to patients undergoing $\mathrm{M}$ alone or $\mathrm{M}$ with any reconstructive procedure [20,21,23,26-28]. OPS is gaining popularity due to its high aesthetic satisfaction, increased tumor free margin rate, and decreased recurrence rate when compared to other surgical interventions $[1,15,16,22,26,29]$. The lower complication rate offers surgeons one more reason to offer OPS. For patients with large tumors located in the upper inner or lower poles of the breast, OPS is now frequently the recommended option [15]. Conversely, mastectomy procedures encompass extensive tissue removal with the added difficulties of reconstruction and skin expansion. Previously reported analyses, specific for $M+R$, have found associated higher complication rates (up to $40 \%$ ) including early infection, bleeding, wound dehiscence, scar formation, nipple loss, capsular contracture, flap loss and functional impairment [5-8].

Similar to previously conducted retrospective studies, our data demonstrates increased acute complications rates associated with smoking $[5,8,14,25,30,31]$, obesity $[5,8,14,25,30,31]$ and advancing age $[5,8,10,31]$. Our adjusted linear regression analysis found that, when compared to diabetics treated with insulin, non-diabetics and diabetics on oral medication only had a much lower incidence of multiple complications. This is in conflict with other retrospective analyses showing no significant difference between patients with and without glycemic control $[8,25,32]$. Similarly, advancing age is a controversial factor for predicting acute complication rates. When adjusted for confounding health status and comorbidities, elderly patients have an increased likelihood to acquire a complication. These results were consistent with other studies $[8,10,25,30]$. Radiation and chemotherapy were significant predictors in the univariate analysis for multiple complications; however, in adjusted MLR, they were insignificant confounders showing no risk of acute postoperative complication [33].

Our data showed that extended operative times correlated with an additional 25\% per hour increased likelihood of complications $[8,14,31,34]$. Likewise, increasing ASA class doubled or tripled the likelihood for complications, especially wound complications and infections [8,14,31,35]. Although surgeons consider comorbidities to be predictive of complications, there is minimal reliable breast cancer research on how certain patient factors including recent weight loss, type of surgical intervention, PMH of CHF, angina, HTN, COPD, renal failure, and bleeding disorders, affect health outcomes.

In the context of elective surgery, COPD, CHF and recent weight loss have been related to an increased risk of pulmonary complications [36]. Intuitively, cardiovascular comorbidities such as hypertension (HTN), angina and CHF are associated with higher cardiac complications [37,38]. Regarding readmission status following elective surgery, a two-fold increase has been associated 
with CHF [39]. Our research closely parallels the results of previously published data on several risk factors associated with non-cardiac surgeries [36-39]. For example, HTN increased odds two-fold $(p=0.003)$ for cardiac complications as did angina $5 \times(p=0.03)$ and underweight BMI $3 \times(p=0.02)$. Furthermore, wound and infectious complications nearly doubled in patients with comorbidities such as bleeding disorders, COPD, HTN, CHF and angina. Similarly, PMH of renal failure exaggerated renal complications ten-fold ( $p=0.03$ ) as did other comorbidities such as CHF, bleeding disorders and recent weight loss (albeit to a lesser extent). The same comorbidities also increased the likelihood of bleeding complications.

Identifying and quantifying these comorbidities preoperatively may allow for better stratification of patient risk and better matching of patients with different operative procedures in order to lower post-operative morbidity [40]. It is logical to adhere to published guidelines on the classification and management of comorbid illnesses prior to surgery: optimizing COPD patients according to GOLD guidelines [41,42], cardiovascular optimization of HTN, CHF, and angina according to the 2014 ACC/AHA guidelines [43], and renal management according to guidelines from the Kidney Disease: Improving Global Outcomes (KDIGO) organization or American Society of Nephrology in order to minimize acute kidney injury post-operatively [44-46].

Return to the OR is a factor in large part under a surgeon's control, done primarily for close/positive surgical margins or due to complications (current rate $=5 \%$; see Table 2). Following this review, and with enhanced knowledge of factors predictive of complications, appropriate action can be taken to further diminish them. Our predictors should allow surgeons to better consent patients and identify those at particular risk for specific complications. Patients with any drains or wound vacuum device placement are more likely to be at risk for a post-operative complication and should be counseled preoperatively We should always try to keep operative time to a minimum (when feasible) and recommend treating patients in the outpatient setting, as both factors were associated with fewer complications across all category types. Interestingly, despite the additional surgery, OPS was associated with the lowest complication rate (other than PM alone), and this factor should be considered in determining the optimal surgical approach for any patient considering mastectomy or mastectomy with reconstruction.

There were several limitations in this study. Oncologic factors, such as tumor size, preoperative nodal involvement, and stage, were not included in the dataset, thereby precluding us from determining their role in deciding the choice of surgical intervention. Interpretation of NSQIP database based on appropriate coding can be problematic, especially with the growing number of NSQIP and CPT codes for OPS. OPS as a reconstructive technique has gained popularity, but CPT codes may vary significantly from one institution to another. We used a coding protocol similar to one used at our institution-as OPS has no specific individual CPT code. Also, long term complications were not recorded in the NSQIP database, thereby potentially skewing the final data. Finally, certain comorbidities such as bleeding disorder and CHF may correlate with one another, but NSQIP did not provide specific medications like the purpose of blood thinners that may have affected these risks and rates of complications. This study highlights the on-going need for further, prospective studies that include the exact surgical procedure, patient comorbidities, and the concomitant use of chemotherapy and radiation therapy in order to help surgeons identify high-risk patients and lower postoperative complications.

\section{Conclusions}

As surgical interventions evolve according to oncological guidelines, patient preferences and modern reconstructive surgeries, the importance of determining and acknowledging complication rates is critical for every surgeon. This paper summarizes most of the risk factors and complications associated with the different kinds of breast surgery. While PM alone has the lowest complication rate (with positive margin rate not included as a complication), it is interesting to note that OPS offers both a form of breast reconstruction and a lower complication rate than either mastectomy alone or 
mastectomy with reconstruction. This factor should be taken into consideration when counseling patients who require more than a simple partial mastectomy.

Supplementary Materials: The following are available online at http:/ / www.mdpi.com/2072-6694/11/2/253/s1, Figure S1: Methods Schematic, Table S1: CPT Codes use to Categorize Breast Cancer Intervention, Table S2: Data from NSQIQ database. Table S3: Unadjusted Logistical Regression.

Author Contributions: M.M.J. was the lead main author of this project and has focused his current Master's program in research on Breast Cancer Intervention, M.M.J. was involved in Conceptualization, methodology, data curation, resources, software analysis, and lastly writing the original and final Draft preparation; J.J. contributed to the research article as follows: methodology, data curation, validation and writing — original draft preparation; R.G. was involved in supervision, investigation, conceptualization and writing-review \& editing portion; Lastly, A.C. was the principle investigator working closely with M.M.J. research, A.C. contributed to conceptualization, methodology, formal analysis, investigation, writing-reviewing \& editing and was project administrator and supervisor.

Funding: The ACS NSQIP were solely the source of data used for this study, herein; they have not verified and are not responsible for the statistical validity of the data analysis or the conclusions derived by the authors. This project does not represent the views or plans of the ACS or the ACS NSQIP. The described study was supported by the National Center for Advancing Translational Sciences, National Institutes of Health, and Award Number TL1TR002546. The content is solely the responsibility of the authors and does not necessarily represent the official views of the NIH.

Conflicts of Interest: The authors declare no conflict of interest.

\section{Abbreviations}

$\begin{array}{ll}\text { Abbrev. } & \text { Definition } \\ \text { BCT } & \text { Breast Conservative Therapy } \\ \text { IvBC } & \text { Invasive Breast Cancer } \\ \text { DCIS } & \text { Ductal Carcinoma in situ } \\ \text { PM } & \text { Partial Mastectomy } \\ \text { M } & \text { Mastectomy } \\ \text { M + R } & \text { Mastectomy with reconstruction } \\ \text { OPS } & \text { Oncoplastic Surgery } \\ \text { M + I } & \text { Mastectomy with breast prosthesis, delayed-insertion or tissue expander for } \\ \text { M }+ \text { MF } & \text { implant placement } \\ \text { MLR } & \text { Mastectomy with Muscular Flap } \\ \text { R2 } & \text { Multivariable Logistical Regression } \\ \text { ACS } & \text { Linear Regression } \\ \text { NSQIP } & \text { American College of Surgeons } \\ \text { CPT } & \text { National Surgical Quality Improvement Program } \\ \text { ICD-9 } & \text { Current Procedural Terminology } \\ \text { CG } & \text { International Classification of Diseases Ninth Revision }\end{array}$

\section{References}

1. Jonczyk, M.M.; Jean, J.; Graham, R.; Chatterjee, A. Surgical trends in breast cancer: A rise in novel operative treatment options over a 12 year analysis. Breast Cancer Res. Treat. 2018. [CrossRef] [PubMed]

2. Cil, T.D.; Cordeiro, E. Complications of oncoplastic breast surgery involving soft tissue transfer versus breast-conserving surgery: An analysis of the NSQIP database. Ann. Surg. Oncol. 2016, 23, 3266-3271. [CrossRef] [PubMed]

3. Chand, N.D.; Browne, V.; Paramanathan, N.; Peiris, L.J.; Laws, S.A.; Rainsbury, R.M. Patient-reported outcomes are better after oncoplastic breast conservation than after mastectomy and autologous reconstruction. Plast. Reconstr. Surg. Glob. Open 2017, 5, e1419. [CrossRef] [PubMed]

4. Bazzarelli, A.; Zhang, J.; Arnaout, A. Patient-Reported Satisfaction Following Oncoplastic Breast-Conserving Therapy. In Proceedings of the American Society of Breast Surgeons Annual Meeting, Dallas, TX, USA, 13 April 2016. 
5. Carlson, G.W. Trends in autologous breast reconstruction. Semin. Plast. Surg. 2004, 18, 79-87. [CrossRef] [PubMed]

6. Schmauss, D.; Machens, H.G.; Harder, Y. Breast reconstruction after mastectomy. Front. Surg. $2015,71$. [CrossRef]

7. Anker, C.J.; Hymas, R.V.; Ahluwalia, R.; Kokeny, K.E.; Avizonis, V.; Boucher, K.M.; Neumayer, L.A.; Agarwal, J.P. The effect of radiation on complication rates and patient satisfaction in breast reconstruction using temporary tissue expanders and permanent implants. Breast J. 2015, 21, 233-240. [CrossRef] [PubMed]

8. Robertson, S.A.; Jeevaratnam, J.A.; Agrawal, A.; Cutress, R.I. Mastectomy skin flap necrosis: Challenges and solutions. Breast Cancer 2017, 9, 141-152. [CrossRef]

9. Carlson, G.W.; Bostwick, J., 3rd; Styblo, T.M.; Moore, B.; Bried, J.T.; Murray, D.R.; Wood, W.C. Skin-sparing mastectomy. Oncologic and reconstructive considerations. Ann. Surg. 1997, 225, 570-575. [CrossRef]

10. Angarita, F.A.; Acuna, S.A.; Cordeiro, E.; Elnahas, A.; Sutradhar, S.; Jackson, T.; Cil, T.D. Thirty-day postoperative morbidity and mortality in elderly women with breast cancer: An analysis of the NSQIP database. Breast Cancer Res. Treat. 2018, 170, 373-379. [CrossRef]

11. Sotheran, W.J.; Rainsbury, R.M. Skin-sparing mastectomy in the UK-A review of current practice. Ann. R. Coll. Surg. Engl. 2004, 86, 82-86. [CrossRef]

12. O’Brien, W.; Hasselgren, P.O.; Hummel, R.P.; Coith, R.; Hyams, D.; Kurtzman, L.; Neale, H.W. Comparison of postoperative wound complications and early cancer recurrence between patients undergoing mastectomy with or without immediate breast reconstruction. Am. J. Surg. 1993, 166, 1-5. [CrossRef]

13. Services USDoHH. Body Mass Index (BMI); Center for Disease Control and Prevention: Atlanta, GA, USA, 2015. Available online: https://www.cdc.gov/healthyweight/assessing/bmi/index.html (accessed on 1 November 2018).

14. El-Tamer, M.B.; Ward, B.M.; Schifftner, T.; Neumayer, L.; Khuri, S.; Henderson, W. Morbidity and mortality following breast cancer surgery in women: National benchmarks for standards of care. Ann. Surg. 2007, 245, 665-671. [CrossRef] [PubMed]

15. Losken, A.; Styblo, T.M.; Carlson, G.W.; Jones, G.E.; Amerson, B.J. Management algorithm and outcome evaluation of partial mastectomy defects treated using reduction or mastopexy techniques. Ann. Plast. Surg. 2007, 59, 235-242. [CrossRef] [PubMed]

16. Tenofsky, P.L.; Dowell, P.; Topalovski, T.; Helmer, S.D. Surgical, oncologic, and cosmetic differences between oncoplastic and nononcoplastic breast conserving surgery in breast cancer patients. Am. J. Surg. 2014, 207, 398-402. [CrossRef] [PubMed]

17. Axelrod, D.A.; Upchurch, G.R., Jr.; DeMonner, S.; Stanley, J.C.; Khuri, S.; Daley, J.; Henderson, W.G.; Hayward, R. Perioperative cardiovascular risk stratification of patients with diabetes who undergo elective major vascular surgery. J. Vasc. Surg. 2002, 35, 894-901. [CrossRef] [PubMed]

18. American Cancer Society. Breast Cancer Facts \& Figures 2017-2018; American Cancer Society, Inc.: Atlanta, GA, USA, 2017; Available online: https:/ /www.cancer.org/content/dam/cancer-org/research/cancer-factsand-statistics / breast-cancer-facts-and-figures/breast-cancer-facts-and-figures-2017-2018.pdf (accessed on 10 October 2018).

19. Labor USdo. Health Plans \& Benefits: Womens' Health and Cancer Rights; United States department of Labor: Washington, DC, USA, 2018. Available online: https://www.dol.gov/general/topic/health-plans/womens (accessed on 11 December 2018).

20. Bertozzi, N.; Pesce, M.; Santi, P.L.; Raposio, E. Oncoplastic breast surgery: Comprehensive review. Eur. Rev. Med. Pharmacol. Sci. 2017, 21, 2572-2585. [PubMed]

21. Carter, S.A.; Lyons, G.R.; Kuerer, H.M.; Bassett, R.L., Jr.; Oates, S.; Thompson, A.; Caudle, A.S.; Mittendorf, E.A.; Bedrosian, I.; Lucci, A.; et al. Operative and oncologic outcomes in 9861 patients with operable breast cancer: Single-institution analysis of breast conservation with oncoplastic reconstruction. Ann. Surg. Oncol. 2016, 23, 3190-3198. [CrossRef]

22. Haloua, M.H.; Krekel, N.M.; Winters, H.A.; Rietveld, D.H.; Meijer, S.; Bloemers, F.W.; van den Tol, M.P. A systematic review of oncoplastic breast-conserving surgery: Current weaknesses and future prospects. Ann. Surg. 2013, 257, 609-620. [CrossRef]

23. Campbell, E.J.; Romics, L. Oncological safety and cosmetic outcomes in oncoplastic breast conservation surgery, a review of the best level of evidence literature. Breast Cancer 2017, 9, 521-530. [CrossRef] 
24. Manning, A.T.; Sacchini, V.S. Conservative mastectomies for breast cancer and risk-reducing surgery: The Memorial Sloan Kettering Cancer Center experience. Gland. Surg. 2016, 5, 55-62. [CrossRef]

25. Bennett, K.G.; Qi, J.; Kim, H.M.; Hamill, J.B.; Pusic, A.L.; Wilkins, E.G. Comparison of 2-year complication rates among common techniques for postmastectomy breast reconstruction. JAMA Surgery 2018, 153, 901-908. [CrossRef]

26. Chakravorty, A.; Shrestha, A.K.; Sanmugalingam, N.; Rapisarda, F.; Roche, N.; Querci Della Rovere, G.; Macneill, F.A. How safe is oncoplastic breast conservation? Comparative analysis with standard breast conserving surgery. Eur. J. Surg. Oncol. 2012, 38, 395-398. [CrossRef] [PubMed]

27. Asban, A.; Homsy, C.; Chen, L.; Fisher, C.; Losken, A.; Chatterjee, A. A cost-utility analysis comparing large volume displacement oncoplastic surgery to mastectomy with single stage implant reconstruction in the treatment of breast cancer. Breast 2018, 41, 159-164. [CrossRef] [PubMed]

28. Chatterjee, A.; Offodile, A.C.; Asban, A.; Minasian, R.A.; Losken, A.; Graham, R.; Chen, L.; Czerniecki, B.J.; Fisher, C. A cost-utility analysis comparing oncoplastic breast surgery to standard lumpectomy in large breasted women. Adv. Breast Cancer Res. 2018, 7, 14. [CrossRef]

29. Jonczyk, M.M.; Patel, K.; Graham, R.; Naber, S.; Erban, J.; Chen, L.; Chatterjee, A. Does Oncoplastic Surgery Offer Low Positive Margin Rates Using New SSO/ASBrS/ASTRO Margin Guidelines? In Proceedings of the San Antonio Breast Cancer Symposium, San Antonio, TX, USA, 5-9 December 2017. Tufts Medical Center: Poster Presentation.

30. Chang, E.I.; Vaca, L.; DaLio, A.L.; Festekjian, J.H.; Crisera, C.A. Assessment of advanced age as a risk factor in microvascular breast reconstruction. Ann. Plast. Surg. 2011, 67, 255-259. [CrossRef] [PubMed]

31. Cordeiro, E.; Jackson, T.D.; Elnahas, A.; Cil, T. Higher rate of breast surgery complications in patients with metastatic breast cancer: An analysis of the NSQIP database. Ann. Surg. Oncol. 2014, 21, 3167-3172. [CrossRef] [PubMed]

32. Lopez-de-Andres, A.; Jimenez-Trujillo, I.; Hernandez-Barrera, V.; de Miguel-Diez, J.; Mendez-Bailon, M.; de Miguel-Yanes, J.M.; Perez-Farinos, N.; Salinero-Fort, M.A.; Del Barrio, J.L.; Romero-Maroto, M.; et al. Association of type 2 diabetes with in-hospital complications among women undergoing breast cancer surgical procedures. A retrospective study using the Spanish National Hospital Discharge Database, 2013-2014. BMJ Open 2017, 7, e017676. [CrossRef]

33. Tanaka, S.; Hayek, G.; Jayapratap, P.; Yerrasetti, S.; Hilaire, H.S.; Sadeghi, A.; Corsetti, R.; Fuhrman, G. The impact of chemotherapy on complications associated with mastectomy and immediate autologous tissue reconstruction. Am. Surg. 2016, 82, 713-717.

34. Cheng, H.; Clymer, J.W.; Po-Han Chen, B.; Sadeghirad, B.; Ferko, N.C.; Cameron, C.G.; Hinoul, P. Prolonged operative duration is associated with complications: A systematic review and meta-analysis. J. Surg. Res. 2018, 229, 134-144. [CrossRef]

35. Hackett, N.J.; De Oliveira, G.S.; Jain, U.K.; Kim, J.Y. ASA class is a reliable independent predictor of medical complications and mortality following surgery. Int. J. Surg. 2015, 18, 184-190. [CrossRef]

36. Smetana, G.W.; Lawrence, V.A.; Cornell, J.E. Preoperative pulmonary risk stratification for noncardiothoracic surgery: Systematic review for the American College of Physicians. Ann. Intern. Med. 2006, 144, 581-595. [CrossRef] [PubMed]

37. Charlson, M.E.; MacKenzie, C.R.; Gold, J.P.; Ales, K.L.; Topkins, M.; Shires, G.T. Intraoperative blood pressure. What patterns identify patients at risk for postoperative complications? Ann. Surg. 1990, 212, 5675-5680. [CrossRef]

38. Yancy, C.W.; Jessup, M.; Bozkurt, B.; Butler, J.; Casey, D.E., Jr.; Colvin, M.M.; Drazner, M.H.; Filippatos, G.S.; Fonarow, G.C.; Givertz, M.M.; et al. 2017 ACC/AHA/HFSA focused update of the 2013 ACCF/AHA guideline for the management of heart failure: A report of the American College of Cardiology/American Heart Association Task Force on clinical practice guidelines and the Heart Failure Society of America. Circulation 2017, 136, e137-e161. [CrossRef] [PubMed]

39. Hammill, B.G.; Curtis, L.H.; Bennett-Guerrero, E.; O'Connor, C.M.; Jollis, J.G.; Schulman, K.A.; Hernandez, A.F. Impact of heart failure on patients undergoing major noncardiac surgery. Anesthesiology 2008, 108, 559-567. [CrossRef] [PubMed]

40. Smit-Fun, V.; Buhre, W.F. The patient with chronic heart failure undergoing surgery. Curr. Opin. Anaesthesiol. 2016, 29, 391-396. [CrossRef] [PubMed] 
41. Diaz-Fuentes, G.; Hashmi, H.R.; Venkatram, S. Perioperative evaluation of patients with pulmonary conditions undergoing non-cardiothoracic surgery. Health Serv. Insights 2016, 9 (Suppl. 1), 9-23. [CrossRef]

42. Bousquet, J.; Clark, T.J.; Hurd, S.; Khaltaev, N.; Lenfant, C.; O’Byrne, P.; Sheffer, A. GINA guidelines on asthma and beyond. Allergy 2007, 62, 102-112. [CrossRef] [PubMed]

43. Fleisher, L.A.; Fleischmann, K.E.; Auerbach, A.D.; Barnason, S.A.; Beckman, J.A.; Bozkurt, B.; Davila-Roman, V.G.; Gerhard-Herman, M.D.; Holly, T.A.; Kane, G.C.; et al. 2014 ACC/AHA guideline on perioperative cardiovascular evaluation and management of patients undergoing noncardiac surgery: Executive summary: A report of the American College of Cardiology/American Heart Association Task Force on practice guidelines. Circulation 2014, 130, 2215-2245. [CrossRef]

44. Hobson, C.; Singhania, G.; Bihorac, A. Acute kidney injury in the surgical patient. Crit. Care Clin. 2015, 31, 705-723. [CrossRef]

45. Mendu, M.L.; Ciociolo, G.R., Jr.; McLaughlin, S.R.; Graham, D.A.; Ghazinouri, R.; Parmar, S.; Grossier, A.; Rosen, R.; Laskowski, K.R.; Riella, L.V.; et al. A decision-making algorithm for initiation and discontinuation of RRT in severe AKI. Clin. J. Am. Soc. Nephrol CJASN 2017, 12, 228-236. [CrossRef]

46. Stevens, P.E.; Levin, A. Evaluation and management of chronic kidney disease: Synopsis of the kidney disease: Improving global outcomes 2012 clinical practice guideline. Ann. Inter. Med. 2013, 158, 825-830. [CrossRef] [PubMed]

(C) 2019 by the authors. Licensee MDPI, Basel, Switzerland. This article is an open access article distributed under the terms and conditions of the Creative Commons Attribution (CC BY) license (http:/ / creativecommons.org/licenses/by/4.0/). 